\title{
Applications of Knoevenagel condensation reaction in the total synthesis of natural products
}

\author{
$\underline{\text { Majid M. Heravi }}{ }^{1}$, Fatemeh Janati $^{2} \&$ Vahideh Zadsirjan $^{1}$ \\ 1- Department of Chemistry, School of Science, Alzahra University, Vanak, Tehran, Iran \\ 2- Department of Chemistry, Payame Noor University, PO Box: 19395-3697, Tehran, Iran
}

\begin{abstract}
The Knoevenagel condensation reaction is a prominent organic reaction commonly being utilized in the total synthesis of natural and biologically potent products as a vital and frequently beginning step. Naturally occurring compounds having complex structures were demonstrated to exhibit significant biological properties. Due to numerous biological potencies, the total syntheses of them has fascinated and attracted much attention of synthetic organic chemists. In this review, we try to highlight the applications of the Knoevenagel reaction as the key step in the total synthesis of biologically active natural products.
\end{abstract}

Keywords: Adduct, Biologically active compounds, Condensation, Knoevenagel reaction, Natural products, Total synthesis 